\title{
Towards real-time analysis of liquid jet alignment in SFX
}

\section{Jaydeep Patel', Adam Round ${ }^{2}$, Andrew Peele ${ }^{3}$, Adrian Mancuso ${ }^{3}$, Brian Abbey ${ }^{1}$}

\author{
${ }^{1}$ La Trobe University; ${ }^{2}$ European XFEL; ${ }^{3}$ Australian Synchrotron (ANSTO);
}

j.patel2@latrobe.edu.au,adam.round@xfel.eu,adrian.mancuso@xfel.eu,peelea@ansto.gov.au,b.abbey@latrobe.edu.au

Serial femtosecond crystallography (SFX) enables the retrieval of the molecular structure of protein molecules at the atomic level through the measurement of large numbers of small crystals intersecting intense X-ray pulses. The method of sample delivery for SFX has a very significant impact on the success (or otherwise) of the experiment since this can impact the signal-to-noise, resolution, and amount of data that can be obtained. In particular, highly efficient sample delivery is critical, since this minimises the amount of X-ray Free Electron Laser (XFEL) beamtime required as well as reducing sample consumption and data volumes. Here we present the results from a series of liquid jet experiments performed at the European XFEL using gas focused liquid injectors, gas virtual dynamic nozzle (GVDN), and double flow focusing nozzles. Although these methods are well-established and used extensively at the European XFEL a major drawback of using these injectors is that over time the jet can become misaligned with the XFEL beam. At present, this requires regular manual monitoring in order to ensure that the relative drift of the jet with respect to the X-ray beam does not become so significant that the beam either 'clips' or misses the jet entirely. Manual adjustment of the liquid jet to ensure alignment with the Xray beam costs the beamline staff time, is prone to errors, and ultimately reduces the amount of useable data that is collected.

In order to address the issue of jet misalignment we present a novel approach to analysing the liquid stream both with ('hit') and without ('miss') intersection by the X-ray beam using machine vision. Optical images from the from the side microscope currently used to monitor the jet are fed into our machine vision algorithm and used to classify the images as either a hit or miss. Currently we are testing the efficacy of the algorithm with a variety of nozzles and jetting conditions. The algorithm will then be incorporated into the control system at the SFX/SPB beamline at the European XFEL where it will be used to generate an 'alignment correction' to the stepper motors controlling the location of the nozzle within the chamber. Via a continuous feedback loop, fine adjustments will be made to the position of the liquid jet ensuring that maximum X-ray beam/liquid jet overlap is achieved. Since this process is fully automated we anticipate that it will result in a larger volume of useful data being collected without requiring any manual intervention. By increasing the efficiency and reducing the per experiment operational cost of SFX at the European XFEL ultimately more experiments can be performed. In addition, via analysis of the feedback metrology we anticipate that optimised nozzle designs and jetting conditions could be achieved further benefitting the end user.

Keywords: Sample delivery, Automation, Machine Vision 